LETTER TO EDITOR

\title{
The ACE2 Receptor in SARS-CoV-2 and HCoV-NL63: Potential Relationship with Kawasaki Disease in Children?
}

\author{
Ruth E Propper, PhD* \\ Montclair State University, Montclair, USA
}

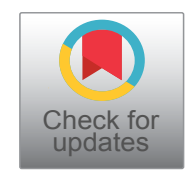

*Corresponding author: Ruth E Propper, PhD, Montclair State University, 1 Normal Avenue, 225 Dickson Hall Montclair, NJ 07043, USA, Tel: 978-697-7889

Coronavirus disease 2019 (COVID-19), the illness caused by Severe Acute Respiratory Syndrome Coronavirus 2 (SARS-CoV-2), is currently causing a global pandemic [1]. Despite its easy transmissibility and oftentimes severe course in adults, children have been predominantly spared the worst of the disease, tending to suffer only mild illness [2]. However, recently across multiple countries, there have been hundreds of anecdotal reports of Kawasaki, or Kawasaki-like disease, in children, potentially linked to COVID-19, with the uptick severe enough for at least one country to issue a nationwide alert to its physicians [3]. This vulnerability in children makes it imperative to determine if there is a causal connection between this Kawasaki-like illness and COVID-19, and to identify the biological processes involved. Crucially, the only other human coronavirus outside the SARS family that uses the Angiotensin Converting Enzyme 2 (ACE2) receptor for cell entry, HCoV-NL63 [4], has also been associated with Kawasaki disease [5]. The link between Kawasaki and this latter coronavirus is not definitive; nevertheless the similarities between HCoV-NL63 and SARS-CoV-19 in ACE2 affinity and in other cellular characteristics, and that some research indicates a link between Kawasaki and HCoV-NL63, bolsters a causal connection between Kawasaki disease and COVID-19, and offers avenue of investigation of biological mechanisms. Whether ACE2 is involved in the clinical etiology of Kawasaki disease is not known. In order to save lives, it is crucial that the course of COVID-19 in children is thoroughly characterized and underlying biological mechanism determined.

\section{Disclosures}

The author declares no financial associations or other possible conflicts of interest. The information has not been presented previously, and no funding was received.

\section{References}

1. World Health Organization (2020) WHO Director-General's opening remarks at the media briefing on COVID-19- 11 March 2020.

2. Castagnoli R, Votto M, Licari A, Brambila I, Bruno R, et al. (2020) Severe acute respiratory syndrome coronavirus 2 (SARS-CoV-2) infection in children: A systematic review. JAMA Pediatr.

3. (2020) Exclusive: National alert as 'coronavirus-related condition may be emerging in children'.

4. Hofmann HK, Pyrc K, van der Hoek LM, Geier B, Berkhout, et al. (2005) Human coronavirus NL63 employs the severe acute respiratory syndrome coronavirus receptor for cellular entry. Proc Natl Acad Sci USA 102: 7988-7993.

5. Pyc K, Berkhout B, Hoek van der L (2007) The novel human coronaviruses NL63 and HKU1. J Virol 81: 3051-3057. 